\title{
Argumentationsstützende Nebensätze im Deutschen
}

\section{Zum Thema}

Dass es Nebensätze gibt, die zu Argumentationszwecken verwendet werden, ist ein Gemeinplatz, der sich in der Grammatik-Terminologie niederschlägt (hypothetischer Satz, Kausal-, Konsekutiv-, Konzessivsatz). Ob es aber eine einheitliche, aufgrund syntaktisch-pragmatischer Kriterien definierbare Klasse von argumentationsstützenden Nebensätzen gibt, ist nicht klar. In der IDSG (1997) werden die argumentationsstützenden Nebensätze den Satzadverbialsätzen zugerechnet. Die Bezeichnung „Satzadverbialsatz“ verwischt aber die grundlegende Unterscheidung zwischen integrierten und nicht integrierten Nebensätzen, aufgrund derer vier Nebensatztypen auseinandergehalten werden können: Die integrierten, valenzgebundenen Ergänzungssätze (Komplementsätze), die integrierten, nicht-valenzgebundenen Angabesätze (Supplementsätze), die nicht integrierten, Hauptsätzen nicht gleichwertigen Konjunktionalsätze, und die nicht integrierten satzwertigen weiterführenden Relativsätze (Cortès 2000, 2010). Ziel der vorliegenden Arbeit ist es zu zeigen, dass die nicht integrierten, Hauptsätzen nicht gleichwertigen Konjunktionalsätze eine einheitliche Klasse aufgrund ihrer syntaktischen und argumentativen Funktion bilden, was sie nicht nur von den weiterführenden Relativsätzen, sondern vor allem auch von den integrierten, nicht-valenzgebundenen Angabesätzen unterscheidet, obwohl viele Konjunktoren wie wenn, wie usw. sowohl einen Angabesatz als auch einen argumentationsstützenden Nebensatz einleiten können. Bevor ich eine Beschreibung und Klassifizierung der argumentationsstützenden Nebensätze (ab jetzt AsNS abgekürzt) in Abschnitt 3 vorschlage, will ich in Abschnitt 2 eine Definition der argumentativen Funktion im Sprachgebrauch umreißen. Der häufige Gebrauch eines besonderen Korrelattyps, des Resumptivums, bei den AsNS wird in Abschnitt 4 behandelt, und der Aufsatz wird mit einem Fazit in Abschnitt 5 abgeschlossen. 


\title{
2 Mit welchen Mitteln und zu welchem Zweck wird in der Sprache argumentiert?
}

Die Diskrepanz zwischen der logischen Kompetenz, die beim Argumentieren angewandt wird, und den sprachlichen Diskurspraktiken, die zur Überzeugung der Interaktionspartner eingesetzt werden, wurde seit eh und je (Logik vs. Rhetorik) erkannt. Diese Diskrepanz ist selbstverständlich für die vorliegende Untersuchung entscheidend.

\subsection{Schlussregelgeleitete Konklusivitätsmuster vs. Topoi-orientierte Alltagsargumentation}

Die Textlinguistik hat das Argumentieren schon lange als kohärenzstiftende Kraft für einen bestimmten Texttyp anerkannt, der von Langer (1995: 70) folgendermaßen definiert wird:

\begin{abstract}
Die argumentative Themenentfaltung dient der Belegung einer Behauptung (These), die das Thema darstellt, durch bestimmte Daten, die aufgrund der hypothetischen Aussagestruktur „Wenn x und y gelten, dann gilt auch die These z“ im Text eine Einheit bilden, und zwar selbst dann, wenn die sie stützenden Glieder nur implizit realisiert und mitzudenken sind, wie dies in Alltagstexten oft der Fall ist.
\end{abstract}

Von den drei herkömmlich unterschiedenen Vernetzungsmustern (Koordinierungs-, Chronologisierungs- und Konklusivitätsmuster) interessiert uns hier das Konklusivitätsmuster, das von Linke/Nussbaumer/Portmann (1994: 241) wie folgt definiert wird:

Dieser dritte Vernetzungstyp geht auf das Deutungsmuster der Kausalbeziehung (im weitesten Sinne) zurück. Er dominiert in argumentativen und erklärenden Texten und umfasst verschiedene Formen der kausalen Verknüpfung. Zentral sind hier die Ursache-Wirkungbzw. die Grund-Folge-Beziehung, im Weiteren gehören hierher auch die Beziehungen von Konditionalität, Konzessivität etc.

Diese Definition, die vor allem auf die Kausalitätsbeziehungen hinweist, wäre aber nicht vollständig, wenn die der Kausalität anhaftende Zeitlichkeit nicht erwähnt würde:

Dieses (Konklusivitäts)-Modell baut in gewisser Weise auf dem der Chronologisierung auf: Basis der kausalen und konditionalen Verknüpfungen sind Beziehungen der Vor- und Nachzeitigkeit. Im Gegensatz zum Modell der Koordinierung ist das Modell für die Konklusivität deshalb mit einer Zeitachse verbunden. (ebd.: 242) 
Zeitlichkeit hat hier nichts mit etwa der Progression des Textablaufs zu tun; es handelt sich um die unerlässliche Voran-Relation zwischen Ursache/Grund und Wirkung: Unsere Welt ist nämlich so beschaffen, dass die Wirkung der Ursache nicht vorangehen kann. Eher als Vorzeitigkeit ist hier die Voran-Beziehung auf der linearen, schlussgerichteten Achse des Konklusivitätsmusters gemeint, wie es z.B. von Toulmin (1975) modelliert wird.

In Toulmins Modellierung des Konklusivitätsmusters als Struktur alltagsprachlicher Argumentationen, in denen Gründe für Behauptungen, Annahmen, Wertungen etc. angeführt werden, basiert der Argumentationsprozess auf einem zielgerichteten Schlussverfahren A > B > C, und zwar: Von (Ausgangs-) Daten (Position A) aus wird unter Einbezug der in Position B gegebenen allgemeinen Schlussregel die Konklusion C erschlossen. Die Zielrichtung des Schlussprozesses in Toulmins Modell veranschaulicht die grundlegende Kausalitätsrelation und die entsprechende Voran-Orientierung von Position A gegenüber der Position C. Das Argumentationsschema in Toulmins Modell bietet also aufgrund seiner Zielrichtung keineswegs Vorschriften an für die lineare Anordnung der einzelnen Bausteine im Rahmen einer sprachlichen Argumentation, bei der sowohl auf A als auch auf C im Laufe des Diskursaufbaus fokussiert werden kann.

Die vorgestellten Konklusivitätsmodelle erfassen Kausalitätsrelationen im weitesten Sinne (einschließlich Konditionalbeziehungen), aber andere Relationen, die für die sprachliche Alltagsargumentation eine Rolle spielen, bleiben dabei im Dunkeln. Als Beispiel dafür sei der sogenannte „Analogieschluss“ genannt (siehe Beispiel (6) unter Abschn. 3), der von der Logik als Fehlschluss (= „falscher Vergleich“) abgewertet wird, aber aufgrund seines hohen didaktischen Werts und seines häufigen Vorkommens in argumentativen Texten von der Sprachwissenschaft berücksichtigt und als ein gemeinnütziges als Topos bezeichnetes Denkmuster angesehen wird. Auf Beispiele dieser Art und deren Deutung kommen wir in Abschnitt 3 zurück.

Die Existenz von nicht schlussregelgeleiteten Argumentationsmustern führt zu einer neuen Definition der Ziele eines argumentationsgestützten Diskurses. In dialogisch orientierten Theorien wird der Argumentationsbedarf als Zeichen einer Konfrontation der Gesprächspartner und das Ziel des Argumentierens in der Bearbeitung divergenter Geltungsansprüche und im Erreichen der Übereinstimmung gesehen. Die Alltagsargumentation kann ,,als ein regelgeleiteter Austausch [...], bei dem Konsens in Bezug auf eine These erzeugt oder verstärkt wird“, betrachtet werden (Meißner/Wyss (Hgg.) 2017: 15). Die (nicht schlussregelgeleiteten) Alltagsargumente, deren Plausibilität von sprach-, kontext- und epochenspezifischen Faktoren abhängt, müssen als solche aufgelistet werden. Angestrebt wird also von der Sprachwissenschaft eine „Typologie inhaltlicher Muster bzw. Topoi von Alltagsargumentation“ (ebd.). 
Eine genaue Untersuchung der in den jeweiligen Argumentationsverfahren verwendeten Sprachmittel findet sich in der Arbeit von Josef Klein (1987), deren Ergebnisse in seinem 2017 erschienenen Aufsatz zusammengefasst werden. Klein (2017) unterscheidet ARGUMENTIEREN und ERKLÄREN-WARUM wie folgt:

ERKLÄREN WARUM ist das Explizieren des Zustandekommens eines Sachverhalts. ARGUMENTIEREN ist das Stützen (Pro-Argumentieren) oder Angreifen (Kontra-Argumentieren) einer problematisierten $\mathrm{u} / \mathrm{o}$ strittigen Position durch Anführen einer oder mehrerer (tatsächlich oder vermeintlich) unstrittigen Positionen. Letztere heißen Argumente oder Gründe. (ebd.: 67)

$\mathrm{Zu}$ betonen ist, dass es sich in der Alltagskommunikation „nicht um einen deduktiven, keine Ausnahme zulassenden Schluss handelt, sondern um einen 'alltagslogischen' Common-Sense-Schluss auf der Basis von Handlungsnormen und/ oder Wahrscheinlichkeiten. Mitunter werden Prämissen als Antecedens (A) und die Konklusion als „Consequens“(C) bezeichnet.“(ebd.: 69). ERKLÄREN-WARUM, ARGUMENTIEREN und FOLGERN weisen verschiedene Komplexitätsunterschiede auf.

ERKLÄREN-WARUM ist kognitiv komplexer als FOLGERN. [...]. ERKLÄREN-WARUM ist zweifach reflexiv: als Sich zurückwenden von C (als „Explanandum“) zu den Gründen, dann als Rückwendung zum - nicht mehr fraglichen - Ausgangspunkt C (als „Explanatum“). [...]Beim ARGUMENTIEREN kommt zum logischen Charakteristikum des Schließens und dem kognitiven der Reflexivität das interaktionale Charakteristikum der Strittigkeit hinzu. (ebd.)

Im Folgenden (Abschn. 3) wird klar werden, wie sich diese Komplexitätsunterschiede in unserer Klassifizierung der argumentationsstützenden Nebensätze niederschlagen.

Was die Alltagsargumentation betrifft, die nicht auf einem Konklusivitätsmuster beruht, schlägt Klein (2017) eine Liste von Topoi vor, über welche Kinder schon im Vorschulalter verfügen. Die Topoi, die für unsere Klassifizierungszwecke eine ausschlaggebende Rolle spielen (Abschn. 3), werden mit den entsprechenden Charakteristika in Tabelle 1 zusammengefasst. 
Tab. 1: Topoi, die in diesem Beitrag verwendet werden (in Anlehnung an Klein 2017), und deren Charakteristika

\begin{tabular}{ll}
\hline Topos-Bezeichnung & Charakteristika \\
\hline $\begin{array}{l}\text { Ursachen-Topos (= Sammelbegriff für das } \\
\text { Spektrum der Kategorisierung kausaler } \\
\text { Faktoren) }\end{array}$ & $\begin{array}{l}\text { Grund/Ursachen im Sinne von auslösenden } \\
\text { Gegebenheiten, Anlässen, Voraussetzungen } \\
\text { u.Ä. }\end{array}$ \\
\hline $\begin{array}{l}\text { Topos der präferierten Alternative (argu- } \\
\text { mentatives Potenzial zur Entwicklung von }\end{array}$ & $\begin{array}{l}\text { Position, die partnerseitigen Ansprüchen oder } \\
\text { Implikationen als Alternative entgegengehalten } \\
\text { Selbstbehauptung und Selbstbestimmung) } \\
\text { wird (Gegenposition zur partnerseitigen }\end{array}$ \\
\hline $\begin{array}{l}\text { Topos der fehlenden oder ungeeigneten } \\
\text { Bedingung (dient zur Rechtfertigung oder } \\
\text { Erklärung vornehmlich der Ablehnung von }\end{array}$ & $\begin{array}{l}\text { Fehlen oder Mängel von Voraussetzungen zur } \\
\text { Implikung partionen (Kontraargumentation im Fokus) }\end{array}$ \\
$\begin{array}{ll}\text { Ansprüchen des Gesprächspartners) } \\
\text { Vergleichstopos (Schluss aus einer } \\
\text { vergleichenden Abwägung, wenn er dem }\end{array}$ & $\begin{array}{l}\text { Im „Topos aus dem Vergleich“ wird unter einem } \\
\text { Erklären oder Rechtfertigen dient) }\end{array}$ \\
\hline
\end{tabular}

Nach der Beschreibung der beiden unterschiedenen Argumentationsmuster muss auf eine grundsätzliche pragmatische Ambiguität hingewiesen werden, die bei der Interpretation der AsNS eine ausschlaggebende Rolle spielt.

\subsection{Pragmatische Ambiguität des Argumentierens in Satz und Text}

Im Satz wie im Text besteht eine grundlegende pragmatische Ambiguität, die für unsere Untersuchung entscheidend ist und sich mit Sweetsers Modell (Sweetser 1990) erfassen lässt. In ihrem Modell unterscheidet Sweetser nämlich drei Referenz-Bereiche: Sachwelt, Denken und Sprechakt, die die Menschen kognitiv analog konzipieren. In allen drei Bereichen sind konditionale oder kausale Beziehungen möglich.

Wenn wir also von einem bewirkten Sachverhalt q auf einen bewirkenden Sachverhalt $\mathrm{p}$ zurückschließen, übertragen wir auf q das Konzept der Ursache, das uns aus dem Welt-Bereich vertraut ist. Allerdings besteht nun die Beziehung nicht mehr primär zwischen zwei Sachverhalten, sondern zwischen zwei Gedanken resp. Schritten in einem Schlussprozess (Peyer 1997: 199).

Eine Äußerung kann zugleich Träger eines Inhalts, Baustein in einem Schlussprozess und Mittel zum Vollzug eines Sprechakts sein. Dabei hängt die 
Wahl der korrekten Interpretation nicht primär von der Form ab, sondern beruht auf ,a pragmatically motivated choice between viewing the conjoined clauses as representing content units, logical entities, or speech acts“ (Sweetser 1990: 78). Eine kausale Relation ist z.B. auf Sachwelt-Ebene zwischen zwei realen Sachverhalten oder Ereignissen (Real-Grund) denkbar, sie kann aber auch als Begründung für die Behauptung einer Annahme oder eines Schlusses auf epistemischer Ebene (Denken/Schließen) (= Erkenntnisgrund) oder als Rechtfertigung einzelner (Sprech-)Handlungen auf Sprechakt-Ebene interpretiert werden. Sweetser (1990) geht davon aus, dass mangels formaler Hinweise die drei kognitiv gleich konzipierten Referenzbereiche aufgrund von pragmatischen Faktoren im Laufe einer kooperativen Interpretationsarbeit richtig analysiert werden können. Wie wir sehen werden, liefert die Unterscheidung gebundene/nicht gebundene Nebensatzstruktur eine wertvolle Interpretationshilfe, wie auch Intonation oder Stellung des Nebensatzes im Satz. Aber der Hörer verfügt selten über eindeutige Marker, wenn es darum geht, zu entscheiden, ob ein Satz auf Sachwelt-Ebene, auf epistemischer Ebene oder auf Sprechakt-Ebene zu interpretieren ist. Für jeden Satz muss der Interpretierende auf den Kontext, die Situation, seine Sprachkompetenz, einschließlich der kommunikativen und kulturellen Kompetenz, usw. Bezug nehmen. Deswegen ist es keineswegs einfach eine klare Klassifizierung der argumentationsstützenden Nebensätze auszuarbeiten, was wir aber trotzdem in Abschnitt 3 versuchen wollen, indem wir für jeden argumentationsstützenden Nebensatztyp die soeben erwähnte pragmatische Ambiguität mit in Kauf nehmen.

\section{Zu einem Inventar der argumentations- stützenden Nebensätze (AsNS)}

Die Annahme einer argumentationsstützenden Funktion für bestimmte deutsche Nebensätze geht davon aus, dass Argumentationsbeziehungen nicht nur zwischen Sätzen, sondern auch innerhalb eines Satzes bestehen können. Der Nebensatz, der darin eingesetzt wird, ist prinzipiell syntaktisch nicht-integriert, dem Hauptsatz aber nicht gleichwertig. Dies wollen wir anhand syntaktischpragmatischer Tests am Beispiel des $d a$-Satzes zeigen, der vorläufig als Prototyp der zu Argumentationszwecken benutzten Nebensätze angesehen werden darf.

(1) Die Kernfunktion von Definitheit ist nicht transphrastischer Natur, da sie als Basiskategorie bereits im infiniten und damit 'subphrastischen' Bereich ihr Funktionspotential entfalten muss. (Leiss 2000: 260) 
Der da-Satz (1) kann nicht im Skopus einer Satznegation, einer Gradpartikel oder eines Modalisators stehen, ist nicht mit einem $w$-Wort (etwa warum) erfragbar und kann auch nicht im Skopus einer Satzfrage stehen, während bei einem gebundenen weil-Angabesatz dieselben Tests alle positiv ausfallen. Wenn der Hauptsatz den Fragemodus (V1?) aufweist, kann der da-Satz, der aufgrund seiner Nicht-Gebundenheit außerhalb des Fragesatzes steht, als Rechtfertigung für die Fragehandlung interpretiert werden, was die IDSG (1997: 2304) als „Moduskommentierung“ analysiert, während ein gebundener weil-Satz, der im Skopus des Gesamtfragesatzes steht, als „Propositionsmodifikator“ fungiert. Die Unterscheidung zwischen dem (nie gebundenen) $d a$-Satz und dem gebundenen weil-Satz kann aufgrund des Modells von Sweetser wie folgt erfasst werden: Der da-Satz referiert immer auf den mentalen Bereich des Denkens, während der gebundene weil-Satz (Angabesatz) immer sachweltbezogen ist. Die nicht gebundenen weilSätze, die als Begründung für das Gesagte oder für den Vollzug bestimmter Sprechhandlungen fungieren (siehe ebd.: 2304-2307, Beispiel Weil du es unbedingt wissen willst, ...), gehören ihrerseits der Sprechaktebene an. Der weil-Satz kann also als Teil eines ERKLÄR-WARUM-Prozesses entweder auf Sachwelt- oder auf Sprechakt-Ebene analysiert werden, während der da-Satz an einem ARGUMENTIEREN-Prozess teilhat und den AsNS eindeutig zugerechnet werden darf. Nach Klein (2017: 70) enthält die im Hauptsatz vertretene These eine gewisse „Strittigkeits-“Komponente, die es mit Stützung des „unstrittigen“ da-Satz-Arguments zu überbrücken gilt.

Die AsNS sind also syntaktisch ungebundene Nebensätze, die aber zusammen mit dem Hauptsatz eine pragmatische Diskurseinheit zu Argumentationszwecken bilden. Aus dem Grammatikalisierungsprozess, den sie durchlaufen haben, resultiert ein charakteristisches syntaktisch-pragmatisches Verhalten, das sie sowohl von den gebundenen Adverbialsätzen (wie oben gesehen) als auch von anderen nicht-gebundenen Nebensätzen wie den weiterführenden Relativsätzen unterscheiden.

Der Versuch, zwei Typen von gebundenen Nebensätzen auf Satz- und Illokutionsebene zu unterscheiden, scheint zum Scheitern verurteilt, denn der Test, der darin besteht, Modalpartikeln in den Nebensatz einzufügen, fällt für alle gebundenen Nebensätze positiv aus, die somit Wurzeleigenschaften aufweisen. Das Auftreten in einem Nebensatz von Modalpartikeln, die „nur beim Vorhandensein illokutiver Kraft lizenziert werden können“, betrachtet Coniglio (2011: 149-153) als Hinweis darauf, dass der nicht gebundene (= ,periphere“) Nebensatz illokutiv eigenständig, d.h. direkt beim Sprecher verankert ist. Nach Haegemann (2002, 2006) und Coniglio (2011) müssen die AsNS als „periphere“ Adverbialsätze (im Gegensatz zu den gebundenen „zentralen“ Adverbialsätzen) und die weiterführenden Relativsätze (wRS) als „periphere“ nicht-restriktive Relativsätze angese- 
hen werden. „Peripher“ und illokutiv eigenständig sind nach dieser Definition alle nicht gebundenen Nebensätze, ob AsNS oder wRS.

Um die AsNS von den wRS zu unterscheiden, muss auf andere Kriterien rekurriert werden. Was sich hier zeigt, ist ein Unterschied nicht in dem illokutiven Potenzial der Nebensätze, sondern in ihren informationsstrukturellen Eigenschaften.

Nach Brandt (1990) können sowohl der ungebundene Nebensatz als auch der Hauptsatz ,als Ausdruck einer selbständigen Informationseinheit betrachtet werden“ (ebd.: 110). Die Informationseinheit ist eine sprachliche Einheit mit eigener Fokus-Hintergrund-Gliederung, die sich pragmatisch textstrukturierend auswirkt.

Die wRS sind keineswegs in die Informationsstruktur des Gesamtsatzes eingegliedert, sondern vermitteln eine Haupt- oder Nebeninformation, je nachdem, ob sie als satzwertige Kommentare oder als Parenthese bzw. Exkurs zu betrachten sind. „Weiterführende RS sind solche, die in Bezug auf die Wichtigkeit der Information mit Hauptsätzen überreinstimmen.“(ebd.: 130).

Die $d a$-Sätze, wie auch alle anderen AsNS, dagegen weisen zwar stets eine eigene Informationsstruktur auf, sind aber vor allem dadurch charakterisiert, dass sie sich in die Fokus-Hintergrund-Gliederung des Gesamtsatzes einordnen und sich als Träger von Hintergrundinformationen definieren lassen. Bei der kommunikativen Gewichtung der Information spielt die Nebensatz-HauptsatzStruktur die ausschlaggebende Rolle: „Bei einem Satzgefüge, das zwei Informationseinheiten vermittelt, signalisiert die Nebensatzform - im Normalfall -, welche Informationseinheit als die weniger wichtige aufgefasst werden soll“ (ebd.: 117).

In den hier beschriebenen Argumentationsmustern ergibt sich eine Gewichtung der Information durch Subordination daraus, dass der Nebensatz die Hintergrundinformation und der Hauptsatz die fokussierte Information trägt. Wie der da-Satz in (1) teilen der hypothetische (2), (3), (4), der konzessive AsNS (5) oder der analogisch konstruierte Satz (6) tatsächlich eine Hintergrundinformation mit, die als Grundlage für das Argumentationsverfahren fungiert und dessen Inhalt gerade deshalb nicht fokussierbar ist. Zu demselben Ergebnis kommt auch Pittner (1999: 236f.).

Für Redder (1990) ergibt sich aus dem Einsetzen eines da-Satzes eine besondere Wissensorganisation: „Die diskursive Leistung der ' $d a$ '-Konstruktion besteht in einer Organisation des verbindlichen Wissens als Ausgangspunkt des darauf aufbauenden, interaktiv thematischen Wissens. [...] Prozedural erfolgt eine sprecherseitige Inanspruchnahme von Wissen als gemeinsamem.“ (ebd.: 200). Im Gegensatz dazu wird im argumentativen wenn-Satz (2), (3) oder V1-Satz (4) eher auf ein nicht-sprecherbasiertes Wissen zurückgegriffen. Hier zeigt sich also eine 
Parallele zu der Beobachtung von Dancygier/Sweetser (2000: 130) für den Gebrauch von if in faktischen Konditionalsätzen: „... if [...] may just acknowledge that this claim is not the speaker's own.“ (Gohl 2000: 22). Dieselbe Feststellung gilt auch für den Gebrauch von wenngleich-Konzessivsätzen (5), während der obwohl-Satz wie der $d a$-Satz ,eine sprecherseitige Inanspruchnahme von Wissen als gemeinsamem“ (Redder (1990: 200) impliziert. Nebensätze mit Verberststellung wie auch die wenn- bzw. wenngleich-Sätze lassen die Frage der Inanspruchnahme des Nebensatzinhalts offen. Auf die Funktion von dann und so in allen Beispielen kommen wir in Abschnitt 4 zurück.

(2) Wenn wir bei der Förderung unserer gemeinsamen wirtschaftlichen Interessen zusammenarbeiten, dann werden wir auch die Barrieren abbauen, die uns seit Jahrhunderten getrennt haben. (Spiegel 98, 17/144 Hume über den Friedensvertrag mit Nordirland)

(3) Wenn dies Wahnsinn ist, so hat es doch Methode. (Shakespeare, Hamlet)

(4) Sollten zu Beginn noch irgendwelche Hemmungen vorhanden gewesen sein, so sind diese schon nach fünf Minuten wie weggeblasen. (St. Galler Tagblatt, 21.1.2011: 51)

In Beleg (1) wird im AsNS ein Pro-Argument eingeführt, während bei (2), (3) und (4) von einer Annahme ausgegangen wird. Im konzessiven Beispiel (5) wird ein Gegenargument eingeführt, das zu einem anderen Schluss führen sollte, als die im Hauptsatz verteidigte These. Das konzessive Satzmuster impliziert die Überwindung des argumentativen Gegensatzes zugunsten des vom Sprecher bevorzugten Schlusses, was durch den Gebrauch der Partikel doch im Hauptsatz bestätigt wird. Dabei wird nicht auf ein schlussregelgeleitetes Konklusivitätsmuster rekurriert, sondern entweder auf das Topos der „Präferierten Alternative“ (= Gegenposition zur partnerseitigen Position) oder auf das Topos „der fehlenden oder ungeeigneten Bedingung“ (= Mangel von Voraussetzungen zur Erfüllung partnerseitiger Ansprüche).

(5) Wenngleich Toulmin eine Pragmatisierung der Argumentationsforschung eingeleitet hat, so basiert sein Schlussschema doch auf Annahmen, die der Situiertheit natürlicher Interaktionen kaum Rechnung tragen. (Meißner/Wyss (Hgg.) 2017: 14)

Als Beispiel für eine auf einem Analogie-Topos aufbauende Konstruktion sei Beleg (6) genannt, wo die Beschreibung eines konkreten Schmerzes die Intensität eines psychologischen Schmerzes versanschaulichen soll. Das Konklusivitäts- 
muster spielt hier keine Rolle, aber der Nebensatzinhalt kann weiter als Träger einer Hintergrundinformation angesehen werden.

(6) Wie eine Wunde erst $\mathrm{zu}$ brennen beginnt, wenn sie verunreinigt wird, so spürt Maria Stuart ihre Niederlage erst, seit man sie mit Hohn vergiftet. (Stefan Zweig, Maria Stuart: 295)

Aufgrund der vorhergehenden Beobachtungen kann zwischen zwei AsNS-Typen unterschieden werden, denjenigen, bei denen im Rahmen eines schlussregelgeleiteten Konklusivitätsmusters argumentiert wird (da-oder wenn-Satz), und denjenigen, die auf Alltagsargumenten und den entsprechenden Topoi beruhen (wenngleich oder wie so-Satz).

Das kontinuierliche, adjazente sodass, „der einzige zentral konsekutive Konnektor“ (Thurmair 2005: 13) leitet einen Nebensatz ein, der als Sonderfall betrachtet werden muss. Er weist nämlich auf einen wichtigen Punkt des Konklusivitätsmusters, die Konklusion, hin, aber er dient nicht dazu, eine Sprechhandlung wie Begründung oder Einräumung zu argumentativen Zwecken $\mathrm{zu}$ inszenieren. Der Sprecher stellt fest, dass zwischen Hauptsatz und (immer nachgestelltem) Nebensatz eine Folgebeziehung besteht, die auf Sachwelt-Ebene (7) situiert werden kann.

(7) Die Karosserie wölbt sich kuppelartig über Fahrer und Beifahrer, senkt sich im Fond allerdings rapide, sodass große Fahrgäste hinten nur sitzen können, wenn sie den Kopf abknicken. (Die Zeit (Online-Ausgabe), 26.6.2003)

(8) Die Sanitätssoldaten schwankten mehr, als sie gingen, so sehr waren sie übermüdet. (Stefan Zweig 1944, Die Welt von Gestern. Erinnerungen eines Europäers: 284)

Darüber hinaus ist es möglich, eine Folgebeziehung anhand zweier aufeinander folgenden V2-Sätze zu formulieren (8), wobei im ersten V2-Satz die Konsequenz und im so-V2-Satz die Ausgangsdaten angegeben werden. Deshalb stimme ich Brandt zu, wenn sie zwischen zwei Typen von Konjunktionalsätzen unterscheidet, denjenigen die „,sich wie die semantisch untergeordneten da-Sätze [und denjenigen, die sich] wie die semantisch nebengeordneten so dass-Sätze verhalten“ (Brandt 1990: 69). In unseren Beispielen (1) bis (6) sind die AsNS alle untergeordnet, aber der sodass-Satz in (7) kann (genauso wie die beiden V2-Sätze in (8)) als nebengeordnet angesehen werden. Sodass-Sätze dürfen aber nicht zu den weiterführenden Relativsätzen hinzugerechnet werden, weil sie einerseits keine kommentierende Funktion erfüllen und weil sie andererseits eindeutig den Konjunktionalsätzen zuzuordnen sind. Die Verbindung von Sachweltbezug und Fortführungsfunktion, die sich in der unabdingbaren Nachstellung widerspie- 
gelt, macht aus dem sodass-Satz eine eigenartige Konstruktion, wie dies schon von Klein (2017: 70) festgestellt wird. Bei FOLGERN wird nur auf einen Teil des Konklusivitätsmusters hingewiesen, ohne dass versucht wird, den Adressaten in das Argumentationsverfahren mit einzubeziehen. Mit dem sodass-Satz wird auf das Ergebnis eines schlussregelgeleiteten Prozesses fokussiert, aber keine argumentative Sprechhandlung realisiert. ${ }^{1}$

Die vorliegende Beschreibung einiger AsNS erhebt keinen Anspruch auf Vollständigkeit. Unsere Ergebnisse fassen wir in den Tabellen 2 und 3 zusammen, angesichts der grundsätzlichen Unterscheidung zwischen schlussregelgeleitetem Argumentationsmuster und Alltagsargumentation aufgrund verschiedener Topoi.

Tab. 2: Schlussregelgeleitetes Argumentationsmuster

\begin{tabular}{lll}
\hline Vollzogene Sprechhandlung & Charakteristika & AsNS-Beispiele \\
\hline Begründung & $\begin{array}{l}\text { sprecherseitige Inanspruch- } \\
\text { nahme von Wissen als } \\
\text { gemeinsamem }\end{array}$ & \\
\hline Hypothese & $\begin{array}{l}\text { Bezug auf hörerbasiertes } \\
\text { Wissen/Distanzierung von }\end{array}$ & \\
& Schon Gesagtem ... dann/wenn ... so \\
& Offenheit des V1-Sacherhalts & V1-Satz ... so \\
\hline
\end{tabular}

Tab. 3: Topoi-gestützte Alltagsargumentation

\begin{tabular}{lll}
\hline Topos-Bezeichnung & Charakteristika & AsNS-Beispiele \\
\hline $\begin{array}{lll}\text { Topos der präferierten } \\
\text { Alternative }\end{array}$ & $\begin{array}{l}\text { Gegenposition zur partner- } \\
\text { seitigen Position }\end{array}$ & Obwohl; obschon, obgleich \\
\hline Topos der fehlenden oder & $\begin{array}{l}\text { Ablehnung von Ansprüchen } \\
\text { des Gesprächspartners }\end{array}$ & $\begin{array}{l}\text { Wenngleich, wenn auch, } \\
\text { selbst wenn auch wenn ... }\end{array}$ \\
ungeeigneten Bedingung & $\begin{array}{l}\text { Referiert unter einem } \\
\text { Vergleichstopos }\end{array}$ & (So) wie ... so-Satz \\
& $\begin{array}{l}\text { gemeinsamen Aspekt auf 2 } \\
\text { andersartige Sachverhalte. }\end{array}$ & \\
\hline
\end{tabular}

1 Der finale damit-Satz darf mit dem sodass-Satz nicht verwechselt werden: Es handelt sich um einen sachweltbezogenen, syntaktisch integrierten Angabesatz, der als eine ERKLÄR-WARUMStruktur zu deuten ist. 
Die AsNS bilden eine eigenständige Klasse von nicht gebundenen (= „peripheren“) Konjunktionalsätzen, die auf Informationsebene die Rolle einer Hintergrundinformation mit argumentativer Stützungsfunktion übernimmt. Die im Hauptsatz vertretene These entspricht der fokussierten Information. Was die Fokus-Hintergrund-Gliederung des argumentativen Gesamtsatzes angeht, so sind Haupt- und Nebensatzinhalt komplementär zueinander, was sie von allen anderen Satzmustern mit nicht gebundenem Relativsatz unterscheidet, wo der Nebensatz keine Hintergrund- sondern eine mehr oder weniger gewichtige Nebeninformation vermittelt.

\section{Resumptiva dann vs. so: Zwei Erkennungsmerkmale für zwei sprachlich belegte Argumentationsmuster}

Die in Abschnitt 3 beschriebenen schlussregelgeleiteten bzw. auf Topoi gründenden AsNS, werden bei Voranstellung relativ häufig mit einem Resumptivum im Hauptsatz wiederaufgenommen, was sie grundsätzlich von allen anderen gebundenen Nebensätzen unterscheidet. Die belegten Resumptiva sind die deiktischen Elemente dann bzw. so, die aufgrund der oben beschriebenen syntaktisch-pragmatischen, topologischen usw. Sprachmittel und bei routinisierter Anwendung in Verbindung mit den mitzudenkenden Topoi eine „Feldtransposition (vom Zeigfeld) in das operative Feld oder Operationsfeld von Sprache“ (Redder 1990: 316) durchgemacht haben, und somit eine spezifische Funktion im Rahmen des mentalen Bereichs der Widerspiegelung von Wirklichkeit (bei Sweetser im Referenzbereich des Denkens) übernehmen.

Die bisherigen Beobachtungen führen uns dazu, die resumptiven Marker dann vs. so als Hinweis des Sprechers auf die gewählte Argumentationsstrategie anzusehen, und generell die Resumptiva als Erkennungsmerkmale für die beiden sich aus Abschnitt 2 und 3 ergebenden Argumentationsmuster. Die These, die in diesem letzten Abschnitt vertreten werden soll, ist folgende: Dann fungiert als Hinweis auf schlussregelgeleitetes Argumentieren aufgrund eines zugrundeliegenden Konklusivitätsmusters und so als Hinweis auf ein Alltagsargumentationsmuster, das sich mit einem Topos erfassen lässt. Dieses Ergebnis ist ohne weiteres mit der Literatur vereinbar (Pasch et al. 2003), im Besonderen mit der Position von Redder (1987), die in der IDSG (1997: 2350) folgendermaßen zusammengefasst wird: 


\begin{abstract}
Die beiden deiktischen Elemente so und dann unterscheiden sich [bei wenn-Sätzen] durchaus in der Art, wie sie auf das im Nebensatz Gesagte rückorientieren. Nach Redder (1987: 318-322) orientiert so auf begriffliche Aspekte der Nebensatzproposition im Vergleich zu Aspekten der Hauptsatzproposition. Dann hingegen leiste ein „lineare Fortentwicklung des hörerseitigen Verstehens und Wissens“ (ebd.: 322), sei es im Sinne einer temporalen Folge (bei temporalen wenn-Sätzen) oder im Sinne einer sachverhaltsbezogenen Folgebeziehung (konditionale wenn-Sätze), oder im Sinne einer Verstehensfolge. (IDSG 1997: 2350)
\end{abstract}

Nur der Bezug auf temporale wenn-Sätze ist hier meines Erachtens fehl am Platz, weil es sich keineswegs um dem mentalen Bereich zugehörige AsNS, sondern um sachweltbezogene Temporalsätze handelt, und vor allem weil dann im temporalen Zusammenhang kein Resumptivum ist, sondern ein Korrelat, das dem temporalen wenn-Satz voran- oder nachgestellt und anhand einer Gradpartikel fokussiert werden kann. Sonst lässt sich ohne weiteres bestätigen, dass das Resumptivum so in allen Varianten von Vergleichstopoi vorkommt, und dass die Verwendung des Resumptivums dann für einen Bezug auf das Konklusivitätsmuster typisch ist. In einem späteren Werk unterstreicht Redder (1990: 39): „Dann [...] leistet eine Neufokussierung in einer linearen vorwärts orientierten Abfolge.“ Dann qualifiziert sie als progredient. Bezüglich so schreibt sie in Fußnote 26: „Im Unterschied dazu habe ich die sprunghafte Folge-Qualität von so als Korrelat von wenn darzulegen versucht (Redder 1987)." Dies kann aufgrund unserer Klassifizierung der AsNS in 3, wo zwischen denjenigen mentalen Operationen, die Anteil an einem Konklusivitäts-Prozess haben, und denjenigen, die Ausdruck von Einschätzungen und Entscheidungen sind, unterschieden wird, etwas einfacher umformuliert werden: In den schlussregelgeleiteten Satzkonstruktionen kann das Resumptivum dann verwendet werden, in den Einschätzungen und Entscheidungen, die auf Topoi beruhen, kommt das Resumptivum so vor. Eine Ausnahme bildet der da-Satz, der generell ohne Resumptivum gebraucht wird, aber ohne Bedenken den schlussregelgeleiteten Satzkonstruktionen zugeordnet werden kann, was sich aufgrund seiner morphologischen Verwandtschaft mit dann leicht nachvollziehen lässt. Die Ergebnisse können in Tabelle 4 zusammengefasst werden:

Tab. 4: Zusammenfassung der Ergebnisse

\begin{tabular}{ll}
\hline $\begin{array}{l}\text { AsNS im Rahmen eines schlussregel- } \\
\text { geleiteten Argumentationsverfahrens }\end{array}$ & $\begin{array}{l}\text { AsNS als Alltagsargumente in } \\
\text { Einschätzungen und Entscheidungen }\end{array}$ \\
\hline Da-Satz & So wie ..., so Analogie \\
Wenn, dann & Wenn, so \\
Falls, dann & Falls, so \\
& V1-Satz, so \\
& Obwohl, obgleich, obschon, so ... doch \\
& Wenn auch, wenngleich, so ... doch \\
\hline
\end{tabular}


Der sodass-Konsekutivsatz wird hier nicht berücksichtigt, weil es sich, wie oben beschrieben, um eine Randerscheinung handelt, mit der keine argumentative Sprechhandlung realisiert wird.

In Abschnitt 2.1 wurde die Verwandtschaft zwischen dem Konklusivitäts- und dem Chronologisierungs-Modell unterstrichen aufgrund der kognitiv verankerten Voran-Beziehung zwischen Ausgangspunkt A und Konklusion C, welche in kausalen und konditionalen Verknüpfungen mitenthalten ist. Es dürfte also nicht überraschen, wenn dann, ein sonst übliches Temporaladverb in der Sachwelt, im mentalen Bereich eingesetzt wird, um eine „Neufokussierung in einer linearen vorwärts orientierten Abfolge“ (Redder 1990: 39) vorzunehmen, da diese „lineare vorwärts orientierte Abfolge“ gerade das dem Gesamtsatz zugrundeliegende Konklusivitätsmuster charakterisiert. Die Verwendung des Resumptivums dann erinnert den Hörer daran, dass eine gewisse Kontiguität und Sukzessivität zwischen A und C im kotopischen Bereich (= im Script oder Frame) des zielgerichteten Schlussverfahrens A > C vorhanden ist und kann als Zeichen für eine grundsätzliche metonymische Relation innerhalb des schlussregelgeleiteten Interpretationsverfahrens angesehen werden. ${ }^{2}$

Mit dem Resumptivum so wird ein ganz anderes Argumentationsverfahren zustande gebracht: Ausgeführt wird die Parallelschaltung zweier asymmetrisch aneinandergereihten Propositionen, die sich heterogenen (allotopischen) Bezugsdomänen zuordnen lassen, was für eine metaphorische Konstruktion typisch ist. Handelt es sich bei der Metonymie um eine der syntagmatischen Achse parallel laufende Relation, so ist bei der Metapher eine der paradigmatischen Achse parallel laufende Relation am Werke, die sich durch eine grundsätzliche Andersartigkeit (in Bonhomme 1987 „Allotopie“ genannt) der verbundenen mentalen Domänen charakterisieren lässt. Die Andersartigkeit des Referenzbezugs (z.B. konkret vs. abstrakt) lässt sich im Analogiemuster (Beleg (6)) verzeichnen. Parallelschaltung und Andersartigkeit der verknüpften Domänen zugleich erzwingen beim Hörer die Suche nach einer „gemeinsamen Einordnungsinstanz“ (Pasch 1983: 104, zitiert von Redder 1990: 266) (Beleg (3), (4), (6) mit so), die sich anhand des Bezugs auf routinisierte Topoi rekonstruieren lässt. In konzessiven Sätzen wird das (auch hier als parallelschaltendes Element fungierende) Resumptivum so fast immer mit der adversativen Partikel doch begleitet, was auf ein eigentümliches Topos hindeutet: Der Sprecher befreit sich von der Forderung, sich nach dem aufgrund des AsNS erwarteten

2 Die Definition der Kotopie im metonymischen Prozess und der Allotopie im metaphorischen findet sich in Bonhomme (1987). Für die Definition der Kontiguitäts- bzw. Similaritätsrelation bei da und so, siehe auch Blühdorn (2003). 
Schluss zu richten, und drückt in aller Deutlichkeit dank der Partikel doch seinen Vorzug für den entgegengesetzten bzw. einen andersartigen Schluss aus, was Klein (1987, 2017) als „Selbstbehauptung“ des Sprechers interpretiert.

Die Opposition dann vs. so in den AsNS kann wie folgt charakterisiert werden: Mit dann wird im kotopischen Rahmen des Konklusivitätsmusters auf eine Kontiguitätsrelation an der linearen zielorientierten Achse zwischen A und C entlang hingewiesen, während mit so auf eine Similaritätsrelation aufgrund einer grundsätzlichen Andersartigkeit (Allotopie) zwischen parallelgeschalteten Domänen hingedeutet wird. Die argumentativen, im Rahmen eines Konklusivitätsmusters schlussregelgeleiteten Satzmuster entsprechen einem metonymischen Pattern, während die Verwendung anderer Topoi in der Alltagsargumentation mit so ein metaphorisches Pattern umsetzt.

\section{Fazit}

Zum Schluss dieser Abhandlung kann Folgendes festgehalten werden: Im Deutschen lässt sich eine Klasse von argumentationsstützenden Nebensätzen (AsNS) definieren, die sich von den gebundenen Angabesätzen dadurch unterscheiden, dass sie nicht sachweltbezogen sind, sondern sich im mentalen Bereich des Denkens auswirken. Der AsNS ist kein Satzglied sondern Teil des rhetorischen Argumentations-Prozesses, dem er aussagemäßig untergeordnet ist. Aufgrund der Polyfunktionalität der Subjunktionen lässt er sich weniger durch die verknüpften Glieder als durch die Art der Verknüpfung definieren. Die pragmatisch einheitliche Satzstruktur mit ungebundenem AsNS basiert entweder auf einer konklusiven Argumentationsstruktur oder auf einem Toposgesteuerten Alltagsargumentationsmuster. Dabei involviert

der Sprecher den Hörer in die mentale Vorgeschichte, die zur Verbalisierung des hauptsächlichen, syntaktisch im Hauptsatz kommunizierten propositionalen Gehalts geführt hat, sei es innerhalb einer Einschätzung, Entscheidung oder eines Schlussverfahrens lokalisiert. (Redder 1990: 264)

\section{Literatur}

Blühdorn, Hardarik (2003): Zur Semantik der Konjunktion als. Paradigmatische und syntagmatische Aspekte. In: Linguistik Online 13. 11-53. (https://bop.unibe.ch/linguistik-online/ article/view/869/1512, Stand: 22.1.2018). 
Bonhomme, Marc (1987): Linguistique de la métonymie. Bern: Lang.

Brandt, Margareta (1990): Weiterführende Nebensätze. Zu ihrer Syntax, Semantik und Pragmatik. (= Lunder Germanistische Forschungen 57). Stockholm: Almqvist \& Wiksell International.

Coniglio, Marco (2011): Die Syntax der deutschen Modalpartikeln: Ihre Distribution und Lizenzierung in Haupt- und Nebensätzen. (= Studia grammatica 73). Berlin: Akademie.

Cortès, Colette (2000): Zur semantisch-pragmatischen Leistung der Hypotaxe im Deutschen. In: Lefevre, Michel (Hg.): Subordination in Syntax, Semantik und Textlinguistik. (= Eurogermanistik 15). Tübingen: Stauffenburg. 85-100.

Cortès, Colette (2010): Correlative markers as phoric “Grammaticalised Category Markers” of subordination in German. (= Studies in Language Companion Series 121). In: Bril, Isabelle (Hg.): Clause linking and clause hierarchy. Amsterdam: Benjamins. 421-447.

Dancygier, Barabara/Sweetser, Eve (2000): Constructions with if, since and because: Causality, epistemic stance, and clause order. In: Couper-Kuhlen, Elisabeth/Kortmann, Bernd (Hgg.): Cause - condition - concession - contrast. Cognitive and discourse perspectives. Berlin/ New-York: De Gruyter. 111-142.

Gohl, Christine (2000): Zwischen Kausalität und Konditionalität. Begründende wenn-Konstruktionen. (= Interaction and linguistic structures 24). In: Deutsche Sprache 30.193-219. (www.inlist.uni-bayreuth.de/issues/24/inlist24.pdf, Stand: 22.1.2018).

Haegeman, Liliane (2002): Anchoring to speaker, adverbial clauses and the structure of CP. In: Georgetown University Working Papers in Theoretical Linguistics 2. 117-180.

Haegeman, Liliane (2006): Conditionals, factives and the left periphery. In: Lingua 116, 10. 1651-1669.

IDSG (1997) = Zifonun, Gisela/Hoffmann, Ludger/Strecker, Bruno (1997): Grammatik der deutschen Sprache. Berlin/New York: De Gruyter.

Klein, Josef (1987): Die konklusiven Sprechhandlungen. Studien zur Pragmatik, Semantik und Lexik von begründen, erklären-warum, folgern und rechtfertigen. Tübingen: Niemeyer.

Klein, Josef (2017): Beginnende Argumentier- und Erklär-Kompetenz (1,9-3,0 Jahre). Vorformen und Topik. In: Meißner, Iris/Wyss, Eva L. (Hgg.). 65-88.

Langer, Gudrun (1995): Textkohärenz und Textspezifizität. (= Europäische Hochschulschriften 152). Bern: Lang.

Leiss, Elisabeth (2000): Artikel und Aspekt: die grammatischen Muster von Definitheit. (= Studia linguistica Germanica 55). Berlin/New York: De Gruyter.

Linke, Angelika/Nussbaumer, Markus/Portmann, Paul R. (1994): Studienbuch Linguistik. Tübingen: Niemeyer.

Meißner, Iris/Wyss, Eva L. (Hgg.) (2017): Begründen - Erklären - Argumentieren. Konzepte und Modellierungen in der Angewandten Linguistik. (= Stauffenburg Linguistik 93). Tübingen: Stauffenburg.

Pasch, Renate (1983): Die Kausalkonjunktionen da, denn und weil. Drei Konjunktionen, drei lexikalische Klassen. In: Deutsch als Fremdsprache 20. 332-337.

Pasch, Renate et al. (2003): Handbuch der deutschen Konnektoren. Linguistische Grundlagen der Beschreibung und syntaktische Merkmale der deutschen Satzverknüpfer (Konjunktionen, Satzadverbien und Partikeln). Berlin: De Gruyter.

Peyer, Ann (1997): Satzverknüpfung - syntaktische und textpragmatische Aspekte. (= Reihe Germanistische Linguistik 178). Tübingen: Niemeyer. 
Pittner, Karin (1999): Adverbiale im Deutschen. Untersuchungen zu ihrer Stellung und Interpretation. (= Studien zur deutschen Grammatik 60). Tübingen: Stauffenburg.

Redder, Angelika (1987): wenn..., so. Zur Korrelation von so. In: Rosengren, Ingrid (Hg.): Sprache und Pragmatik. (= Lunder germanistische Forschungen 55). Stockholm: Almqvist \& Wiksell. 315-326.

Redder, Angelika (1990): Grammatiktheorie und sprachliches Handeln: „denn“ und „da“. (= Linguistische Arbeiten 239). Tübingen: Niemeyer.

Sweetser, Eve (1990): From etymology to pragmatics. Metaphorical and cultural aspects of semantic structure. Cambridge: Cambridge University Press.

Thurmair, Maria (2005): Vergleich und Konsekutivität. In: Deutsche Sprache 33. 1-14.

Toulmin, Stephen (1975): Der Gebrauch von Argumenten. (= Wissenschaftstheorie und Grundlagenforschung 1). Kronberg: Scriptor. 
